\title{
Polyvinylcarbazole films: Applications for chemical sensors
}

\author{
I.V. Kruglenko \\ V. Lashkaryov Institute of Semiconductor Physics, NAS Ukraine \\ 41, prospect Nauky, 03028 Kyiv, Ukraine \\ Phone: (380-44) 525-56-26; e-mail: kruglenko@yahoo.com
}

\begin{abstract}
The adsorption properties of polyvinylcarbazole (PVC) films as sensitive layers of quartz crystal microbalance (QCM) sensors have been studied. Different organic vapors and complex mixture (a cologne) were tested as analytes. PVC films have demonstrated different adsorption properties under different temperatures. This makes it a perspective material for sensor applications.
\end{abstract}

Keywords: sensors, polyvinylcarbazole (PVC), quartz crystal microbalance (QCM).

Manuscript received 08.07.16; revised version received 20.09.16; accepted for publication 16.11.16; published online 05.12.16.

\section{Introduction}

It is impossible to imagine modern development of science without polymers in all their diversity. In recent decades, these materials became of great interest for scientists in the frame of creation of highly selective sensitive layers for sensors that are required in industry, medicine, perfumery, environmental monitoring, food control, etc.

Among sensors based on organic materials, conductive polymers are the most common [1-10]. The advantages of these sensors are above all an opportunity to work at room temperature, easiness of manufacturing and low cost.

Despite the large amount of works in this area, this direction continues to be developed, as evidenced by a large number of articles on this subject, which appear every year.

It was shown [1] that Pd-polyaniline films are highly sensitive and selective to methanol vapors and provide stable signal for a long time. Polyaniline ultrathin layers were prepared using the Langmuir-
Blodgett method, and gas sensitivity of these films to NO was studied [2]. Using the Langmuir-Blodgett methods, thin layers of pure polycarbazole and polycarbazole mixed with octadecylamine were obtained. The sensitivity of these ultrathin films to ammonia was investigated by measuring changes in their electrical resistance. It was found that the LangmuirBlodgett film with pure polycarbazole has higher sensitivity and a faster response as well as recovery to ammonia than the layers of mixed type [3]. In the work [4], the polypyrrole layers that show high sensitivity to ammonia were investigated. Composite polypyrrolepoly(vinyl alcohol) layers were prepared using the electrochemical method and were studied at various concentrations in vapors of ethanol [5]. Nanocomposite of copper/polyaniline was used as a sensor for chloroform. The authors believe that sensitive mechanism mainly includes adsorption-desorption of chloroform on a metal surface of clusters [6]. The polymer sensors were placed into vapors of different gases: o-xylene, n-butane, benzene, methanol, n-heptane, and 3-pentanone. Each sensor was subjected to repeated 


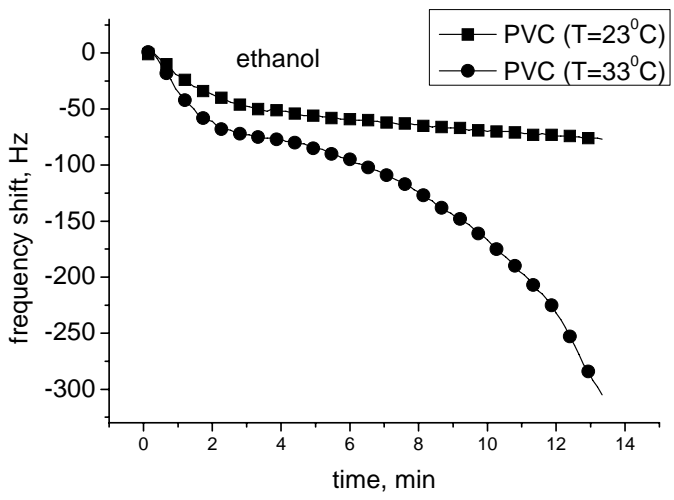

a)

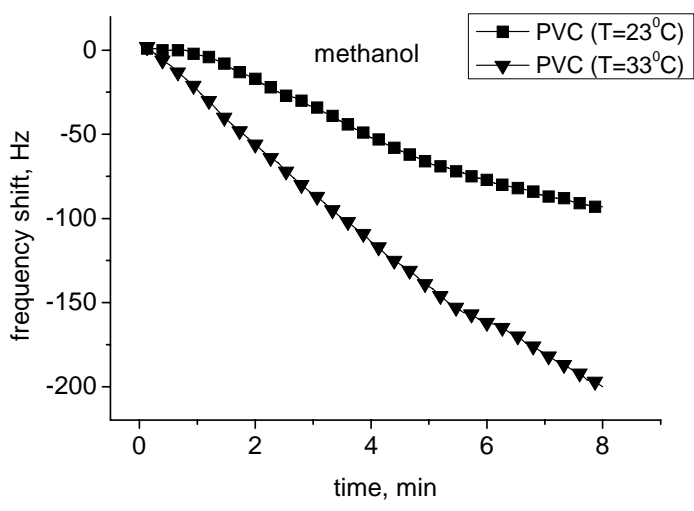

b)

Fig. 1. Responses of QCM sensors based on PVC to saturated vapors of ethanol (a), methanol (b) at different temperatures.

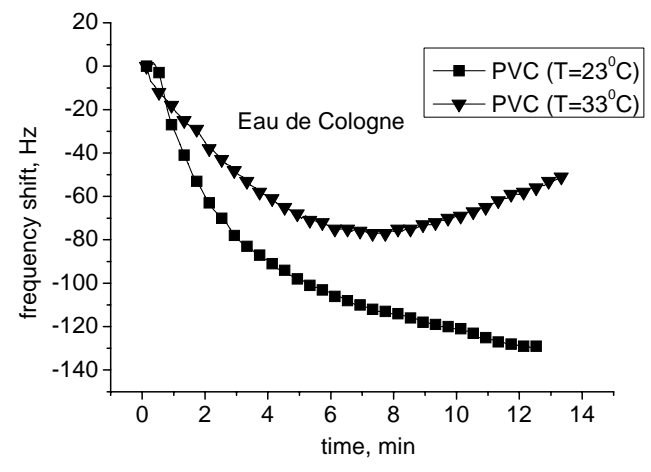

a)

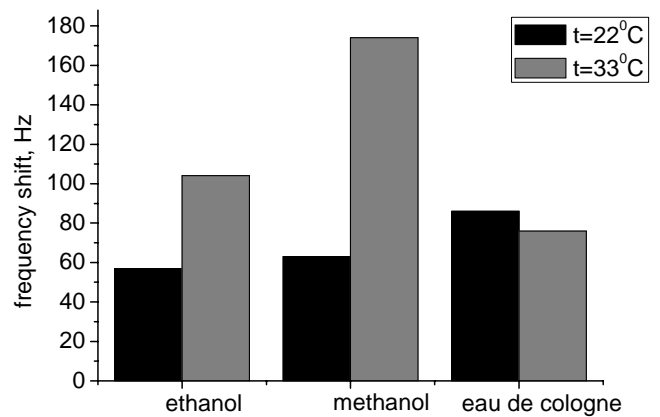

b)

Fig. 2. Responses of QCM sensors based on PVC to Eau de Cologne (a); diagram showing the amplitude of responses of the same sensor to alcohols and cologne for two temperatures (b).

exposure in these vapors at various concentrations. Analysis of the obtained data has shown that the sensitivity depends on the chemical nature of both the sensor and studied vapors as well as duration of exposure in vapors [7].

In this paper, we consider the adsorption properties of thermally deposited polyvinylcarbazole (PVC) films.

\section{Experimental procedure}

In general, today we know quite a number of methods for forming thin film coatings in a wide range of thicknesses - from a few nanometers to hundreds of micrometers. It is interesting to note that along with traditional chemical methods of creating the coatings in the form of thin films, classic methods of microelectronic technology (namely, thermal evaporation in vacuum with subsequent condensation on the respective substrates) are widely used to obtain stable coatings with desired physical and chemical parameters. Polymer (polyvinylcarbazole (from Aldrich)) films were deposited using thermal evaporation in vacuum directly on the electrode of quartz crystal microbalance (QCM).

The QCM sensor operation principle is measurement of variation of the resonant oscillation frequency of a piezoelectric crystal caused by change of its mass due to analyte molecules adsorption onto the sensitive layer surface. The responses of a QCM without sensitive coating to all analytes under investigation did not exceed $10 \mathrm{~Hz}[11,12]$.

Our measurements with the QCM technique were made as follows. The sensors were placed in a measuring flow-type cell and were held in argon flow until stabilization of the quartz resonators frequency (deviation of $\pm 1 \mathrm{~Hz}$ ). Then analyte vapor in argon passed through the cell with a constant flow-rate of about $60 \mathrm{ml} / \mathrm{min}$. The analytes were ethanol, methanol and perfume (Ukraine). The concentrations of analytes were close to the saturation level.

The experiments were performed in a thermostatic chamber where the sensor array and studied samples are located [13]. The temperature in the experiment was kept as 23 and $33^{\circ} \mathrm{C}$. 


\section{Results and discussion}

In this paper, polymer layers of PVC were prepared using the method of vacuum deposition of films. Using the unique chemical and physical properties of this polymer, we studied the influence of vapors of different nature on the surface of QCM with sensitive polymer layer. Characteristics of gas sensors are defined by physical and chemical properties of their sensor elements that, in its turn, depend on the crystal structure, composition and adsorption properties of the sensitive layers in the sensor array. Changing the temperature during experiments, we changed the adsorption properties of the sensor surfaces.

The analysis of sensor responses allows to estimate kinetic curves for all the types of samples. At room temperature, ethanol shows monotonic curves during the whole experiment, methanol - first shows a small plateau that goes also down to the monotonic curves. Fig. 1 shows the influence of temperature on polymer layers when adsorbing alcohol and methanol. As seen from the results obtained, the increase in the temperature by ten degrees $\left(33^{\circ} \mathrm{C}\right)$ leads to change of the form of the response curves when adsorbing ethanol - the sensitivity of polymer layers of quartz crystal and kinetic behavior of the curves are changed. When temperature increases from 23 to $33{ }^{\circ} \mathrm{C}$ much higher response on both ethanol and methanol is observed (2.5 ...5 times).

For experimental researches, we also selected a more complex analyte - Eau de Cologne - a multicomponent mixture containing water-alcohol solution, essential oils, terpenes, alcohols, aldehydes, ketones, menthol, camphor, acetic acid. The evaporation temperatures of different components in this mixture differ and this fact affects the sensor response character. As seen from the figure, with increasing the temperature to $33^{\circ} \mathrm{C}$, a dramatic change in the kinetic of curves takes place when adsorbing cologne.

The determined properties of thermally deposited films of PVC polymer, namely, changing the sensitivity to vapors of alcohol and perfume when changing the temperature, cause considerable interest to this material in terms of its use in arrays of sensors to create devices of the "electronic nose" type.

\section{Conclusions}

Thus, these results indicate that the structures based on quartz/silver/polymer (QSM) can be used in sensor arrays for the analysis of multi-component mixtures based on alcohol, which is important for the chemical and pharmaceutical industry, for production of alcoholic beverages and to environmental monitoring. The temperature can serve as an additional factor affecting the functionality of these sensors.

\section{Acknowledgements}

The author thanks Dr D. Grynko for providing the polyvinylcarbazole samples for researches.

\section{References}

1. A.A. Athawale, S.V. Bhagwat, P.P. Katre, Nanocomposite of Pd-polyaniline as a selective methanol sensor // Sensors and Actuators B, 114, p. 263-267 (2006).

2. D. Xie, Y.D. Jiang, W. Pan, D. Li, Z.M. Wu, Y.R. Li, Fabrication and characterization of polyaniline-based gas sensor by ultra-thin film technology // Sensors and Actuators B, 81, p. 158164 (2002).

3. V. Saxena, S. Choudhury, S.C. Gadkari, S.K. Gupta, J.V. Yakhmi, Room temperature operated ammonia gas sensor using polycarbazole Langmuir-Blodgett film // Sensors and Actuators B, 107, p. 277-282 (2005).

4. N.T. Kemp, A.B. Kaiser, H.J. Trodahl, B. Chapman, R.G. Buckley, A.C. Partridge, P.J.S. Foot, Effect of ammonia on the temperaturedependent conductivity and thermopower of polypyrrole // J. Polym. Sci. B, 44, p. 1331-1338 (2006).

5. C.W. Lin, B.J. Hwang, C.R. Lee, Characteristics and sensing behavior of electrochemically codeposited polypyrrole-poly(vinyl alcohol) thin film exposed to ethanol vapors // J. Appl. Polym. Sci. 73, p. 2079-2087 (1999).

6. S. Sharma, C. Nirkhe, S. Pethkar, A.A. Athawale, Chloroform vapour sensor based on copper/polyaniline nanocomposite // Sensors and Actuators B, 85, p. 131-136 (2002).

7. M.C. Gallazzi, L. Tassoni, C. Bertarelli, G. Pioggia, F. Di Francesco, E. Montoneri, Poly(alkoxy-bithiophenes) sensors for organic vapours // Sensors and Actuators B, 88, p. 178-189 (2003).

8. V. Syritski, J. Reut, A. Öpik, K. Idla, Environmental QCM sensors coated with polypyrrole // Synth. Met. 102, p. 1326-1327 (1999).

9. A.C. Partridge, M.L. Jansen, W.M. Arnold, Conducting polymer-based sensors // Mater. Sci. Eng. C, 12, p. 37-42 (2000).

10. G.E. Collins, L.J. Buckley, Conductive polymercoated fabrics for chemical sensing // Synth. Met. 78, p. 93-101 (1996).

11. I. Kruglenko, B. Snopok, Non-exponential relaxations in sensor arrays: forecasting strategy for electronic nose performance // Sensors and Actuators B: Chem. 106, p. 101-113 (2005).

12. B.A. Snopok, I.V. Kruglenko, Multisensor systems for chemical analysis: state-of-the-art in Electronic Nose technology and new trends in machine olfaction // Thin Solid Films, 418(1), p. 21-41 (2002).

13. Julia Burlachenko, Ivanna Kruglenko, Boris Snopok, Krishna Persaud, Sample handling for electronic nose technology: State of the art and future trends // Trends in Analytical Chemistry, 82, p. 222-236 (2016).. 